\title{
Improved convergence of Complex Langevin simulations
}

\author{
Felipe Attanasio ${ }^{1,2}$ and Benjamin Jäger ${ }^{3,4, \star}$ \\ ${ }^{1}$ Department of Physics, University of Washington, Box 351560, Seattle, WA 98195, USA \\ ${ }^{2}$ Department of Physics, College of Science, Swansea University, Swansea SA2 8PP, United Kingdom \\ ${ }^{3}$ CP3-Origins \& Danish Institute for Advanced Study, Department of Mathematics and Computer Science, \\ University of Southern Denmark, 5230 Odense M, Denmark \\ ${ }^{4}$ Institute for Theoretical Physics, ETH Zürich, CH-8093 Zürich, Switzerland
}

\begin{abstract}
The sign problem appears in lattice QCD as soon as a non-zero chemical potential is introduced. This prevents direct simulations to determine the phase structure of the strongly interacting matter. Complex Langevin methods have been successfully used for various models or approximations of QCD. However, in some scenarios it converges to incorrect results. We present developments of our new method that helps to improve the convergence by keeping the system closer to the SU(3) manifold and discuss preliminary tests and results.
\end{abstract}

\section{Introduction}

Sign problems have been found, and obstructed progress, in many areas of physics. One of the most famous examples is QCD with non-vanishing Baryon chemical potential in Euclidean spacetime. Here the fermion determinant, which appears after integrating out the fermionic degrees of freedom, becomes complex and thus prevents direct simulation via Monte Carlo methods. Complex Langevin simulations offer an alternative to study complex-valued Euclidean path integrals. It has been shown that Complex Langevin methods are applicable even when the sign problem is severe [1-5]. The technique is based on the principle of stochastic quantisation and has been proposed decades ago by Klauder and Parisi [6-10]. More recently, the development of gauge cooling [11, 12] has enabled complex Langevin simulations of QCD with heavy quarks [13, 14] and light quarks [2, 3, 15]. Nevertheless, simulations with smaller gauge couplings, typically below $\beta \sim 5.8$, do not converge to the correct results. To tackle this issue we have introduced an additional force, named Dynamic Stabilisation [16], which is expected to vanish in the continuum limit. In the following we present an update on our studies of dynamic stabilisation for QCD in the limit of heavy quarks, and apply it also to the $\mathrm{XY}$ model at finite chemical potential [17].

\section{Complex Langevin simulation and Dynamic Stabilisation}

The (remaining) degrees of freedoms after integrating the fermions out, i.e. the SU(3) gauge links $U_{x, \mu}$, are evolved for a small time step $\varepsilon$ along a fictitious time dimension, known as the Langevin

^ Speaker, e-mail: jaeger@imada.sdu.dk 
time $\theta$, using [18]

$$
U_{x, \mu}(\theta+\varepsilon)=\exp \left[\mathrm{i} \lambda^{a}\left(\varepsilon K_{x}^{a}+\sqrt{\varepsilon} \eta_{x, \mu}^{a}\right)\right] U_{x, \mu}(\theta),
$$

where $\lambda^{a}$ are the Gell-Mann matrices, $\eta_{x, \mu}^{a}$ are white noise fields and the drift $K_{x, \mu}^{a}$ is given by

$$
K_{x, \mu}^{a}=-D_{x, \mu}^{a} S, \quad \text { where } \quad D_{x, \mu}^{a} f(U)=\left.\frac{\partial}{\partial \alpha} f\left(e^{i \alpha \lambda^{a}} U\right)\right|_{\alpha=0} .
$$

If the drift becomes complex, the Langevin equation naturally extends into the larger gauge group of $\operatorname{SL}(3, \mathbb{C})$. The action $S$ contains the Yang-Mills plaquette action for the gluons and the fermion determinant $\operatorname{det} D$, representing the quarks degrees of freedom. The effective action can be written as

$$
S=S_{Y M}-\ln \operatorname{det} D .
$$

For simplicity, we ignore issues arising from meromorphic drifts originating from the logarithm of the fermion determinant, but refer the reader to the discussions in [19-23]. The fact that $\operatorname{SL}(3, \mathbb{C})$ is not a compact group allows for runaway trajectories. This is usually monitored by the so-called unitarity norm,

$$
d=\frac{1}{3 V} \sum_{x, \mu} \operatorname{Tr}\left(U_{x, \mu} U_{x, \mu}^{\dagger}-1\right)^{2}
$$

where $V$ is the lattice $4-$ volume, which measures the distance to the SU(3) manifold. It is known that large unitarity norms lead to incorrect results [14]. Gauge cooling has been constructed to keep $d$ small, but is ineffective in some situations. As proposed in [16, 24], we modify the Langevin drift

$$
K_{x, \mu}^{a} \rightarrow-D_{x, \mu}^{a} S+\mathrm{i} \alpha_{\mathrm{DS}} M_{x}^{a}
$$

by adding a term that only acts in the non-SU(3) directions and has a restoring character, i.e. it will reduce the distance to the $\mathrm{SU}(3)$ manifold. A possible choice for the force is given by

$$
M_{x}^{a}=i b_{x}^{a}\left(\sum_{c} b_{x}^{c} b_{x}^{c}\right)^{3}, \quad \text { where } \quad b_{x}^{a}=\operatorname{Tr}\left[\lambda^{a} \sum_{v} U_{x, v} U_{x, v}^{\dagger}\right] .
$$

The "strength" of this force can be changed by modifying the control parameter $\alpha_{\mathrm{DS}}$. By construction, the force $M_{x}^{a}$ grows rapidly with the distance to the SU(3) manifold, $M \sim d^{7}$. Since it has been previously shown that $d$ should be kept significantly smaller than one $(d \sim 0.03$ has been taken as a conservative threshold) [14], only sufficiently large control parameters have a non-trivial effect. It is important to note that DS will, for very large $\alpha_{\mathrm{DS}}$, effectively re-unitarise the theory and thus produce incorrect results.

The optimal choice is in the intermediate regime, where the overall distribution of the drifts is narrower and the observables are least sensitive to the control parameter $\alpha_{\mathrm{DS}}$. This behaviour can be seen in Figure 1 for the average Polyakov loop in the HDQCD model, which will be explained below. The figure shows results where the gauge links have been periodically reunitarised, complex Langevin simulations with gauge cooling (GC), and simulations using GC and DS. Two separate analyses have been carried out with the data from the GC runs: in one of them all data points after thermalisation have been considered, while in the other the points after the unitarity norm reached our threshold of $d \sim 0.03$ have been disconsidered. The GC data from the first analysis is known to be incorrect and is shown for comparison.

Gauge cooling is known to produce reliable results when the unitarity norm is small. The agreement with DS simulations for certain intervals of $\alpha_{\mathrm{DS}}$ is evidence that DS succeeds in keeping large explorations of $\operatorname{SL}(3, \mathbb{C})$ under control. 


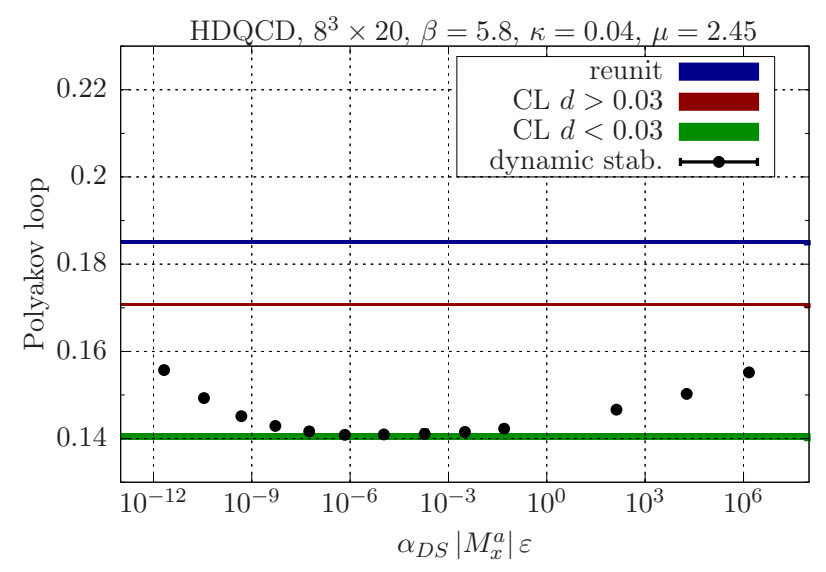

Figure 1. Expectation value of the Polyakov loop as a function of the control parameter $\alpha_{\mathrm{DS}}$. We also show results from simulations with gauge cooling (GC) at different cutoffs for the unit. norm, and with real Langevin.

\section{HDQCD}

The heavy dense approximation of QCD (HDQCD) is an approximation for very large quark masses. Using the hopping parameter expansion and dropping all terms beyond the static limit lead to a significant simplification of the fermion determinant: It can be written solely in terms of the Polyakov loop $P_{\vec{x}}$ and its inverse

$$
\operatorname{det} D(\mu)=\prod_{\vec{x}} \operatorname{det}\left(1+h \mathrm{e}^{\mu / T} P_{\vec{x}}\right)^{2} \operatorname{det}\left(1+h \mathrm{e}^{-\mu / T} P_{\vec{x}}^{-1}\right)^{2}, \quad \text { where } \quad h=(2 \kappa)^{N_{\tau}},
$$

and

$$
P_{\vec{x}}=\prod_{\tau=0}^{N_{\tau}-1} U_{(\vec{x}, \tau), \hat{4}},
$$

with $N_{\tau}$ being the lattice extent in the temporal direction. This simplification allows for quick studies of the effect of Dynamic Stabilisation (DS). Here, we look in particular at the distribution of the drifts, which can be considered as a criterion for convergence [25, 26]. Figure 2 shows a histogram of the imaginary part of the Langevin drift, which has been averaged over the four directions. Larger values for $\alpha_{\mathrm{DS}}$ result in more localised distributions for the Langevin forces and thus improving the overall convergence. Figure 3 shows the equivalent distribution for the SU(3) part of the Langevin drifts. The histogram with $\alpha_{\mathrm{DS}}=1$ is noticeably different, which indicates that indirect effects from the nonSU(3) drifts cause changes to the distribution. For larger values, the real part of the drifts are identical and unaffected by the additional term. If we expand the DS drift in terms of the lattice spacing, using a naïve description of the gauge links

$$
U_{x, \mu}=\exp \left[\mathrm{i} a \lambda^{a}\left(A_{x, \mu}^{a}+\mathrm{i} B_{x, \mu}^{a}\right)\right],
$$

we find that the additional drift formally vanishes in the continuum limit. This is however not a formal proof, which is significantly harder to do, since we cannot rewrite the DS term originating from an action principle. Figure 4 shows the histograms of the DS drift, $M_{x}^{a}$, for three values of the gauge coupling. The counts of larger drifts can be seen to diminish as the continuum limit is approached, thus indicating that the DS force becomes trivial for large $\beta$, as desired. 


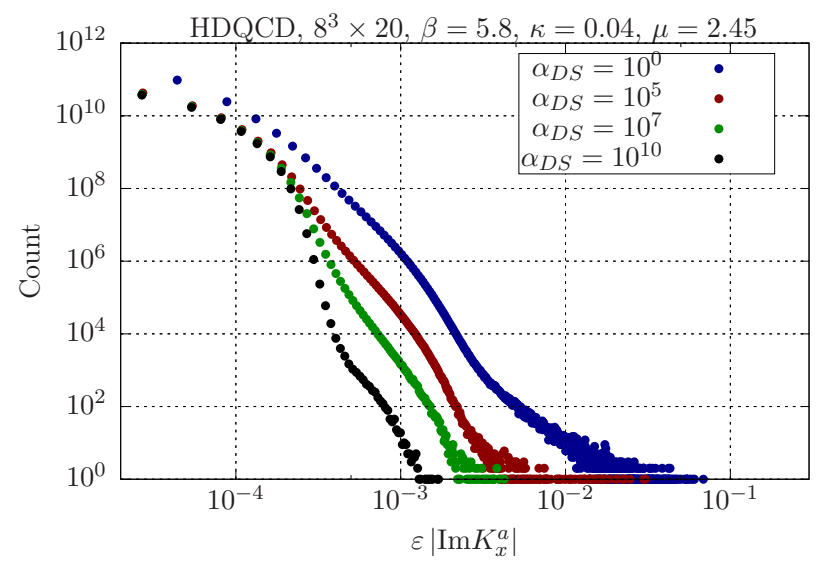

Figure 2. Histograms of the imaginary part of the drift (non-SU(3) part) as function of the magnitude $\varepsilon\left|\operatorname{Im} K_{x}^{a}\right|$ for four values of the control parameter $\alpha_{\mathrm{DS}}$.

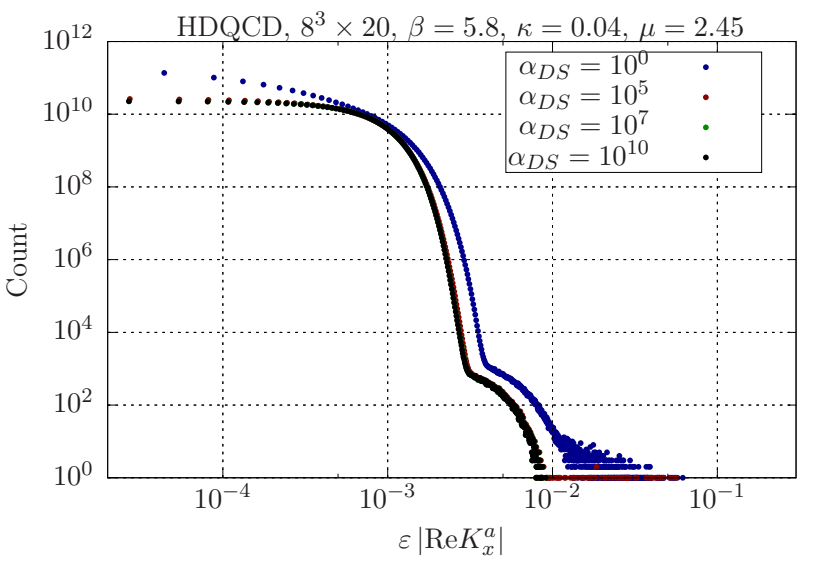

Figure 3. Histograms of the real part of the drift (SU(3) part) as function of the magnitude $\varepsilon\left|\operatorname{Re} K_{x}^{a}\right|$ for four values of the control parameter $\alpha_{\mathrm{DS}}$.

\section{XY Model}

The three-dimensional XY model at finite density, whose action is given by

$$
S=-\beta \sum_{x} \sum_{v=0}^{2} \cos \left(\phi_{x}-\phi_{x+\hat{v}}-\mathrm{i} \mu \delta_{v, 0}\right),
$$

has been studied using complex Langevin simulations [17]. It was concluded that in the disordered phase complex Langevin simulations fail to reproduce the correct results, which can be obtained using a dual, sign-problem-free, world-line formulation [27, 28]. In the following, we report on our tests of a variant of Dynamic Stabilisation applied to the $\mathrm{XY}$ model ${ }^{1}$. The equivalent of the unitarity norm in

\footnotetext{
${ }^{1}$ We thank Gert Aarts for suggesting this.
} 


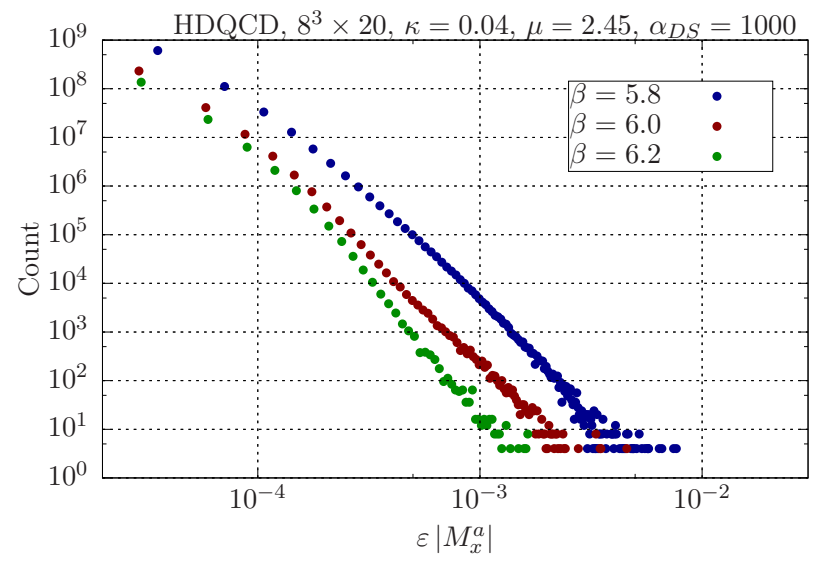

Figure 4. Histogram of the added DS force for three different values of the gauge coupling, keeping other simulation parameters fixed.

this context is simply given by the imaginary part of the complexified scalar field $\phi$, leading to the DS force

$$
K_{\mathrm{DS}} \rightarrow-D_{x, \mu}^{a} S+\mathrm{i} \alpha_{D S}\left[\operatorname{Im} \phi_{x}\right]^{7}
$$

This is, however, a naïve implementation of a DS force, which might be not ideal. Further studies exploring alternative definitions are ongoing. Figure 5 shows the action density $S / \Omega$ as a function of

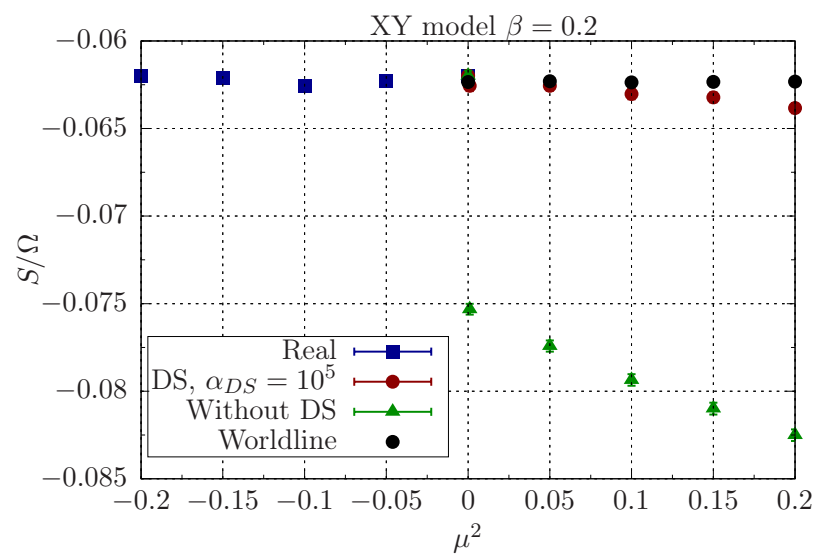

Figure 5. The action density $S / \Omega$ as a function of the chemical potential for the XY model in the disordered phase with $\beta=0.2$. The results for the worldline formulation have been taken from [17].

the chemical potential. With imaginary chemical potentials, i.e. $\mu^{2}<0$, the theory is sign-problemfree and thus real Langevin simulations are applicable and used. For real chemical potentials we compare simulations with and without Dynamic Stabilisation. Standard complex Langevin for $\mu \neq$ 0 shows a significant deviation from the correct results. Adding an additional drift as in Eq. 11 leads to a significant improvement, which however still shows a small, but significant deviation from 
the worldline results, in particular for larger chemical potentials. Figure 6 shows a variation of the

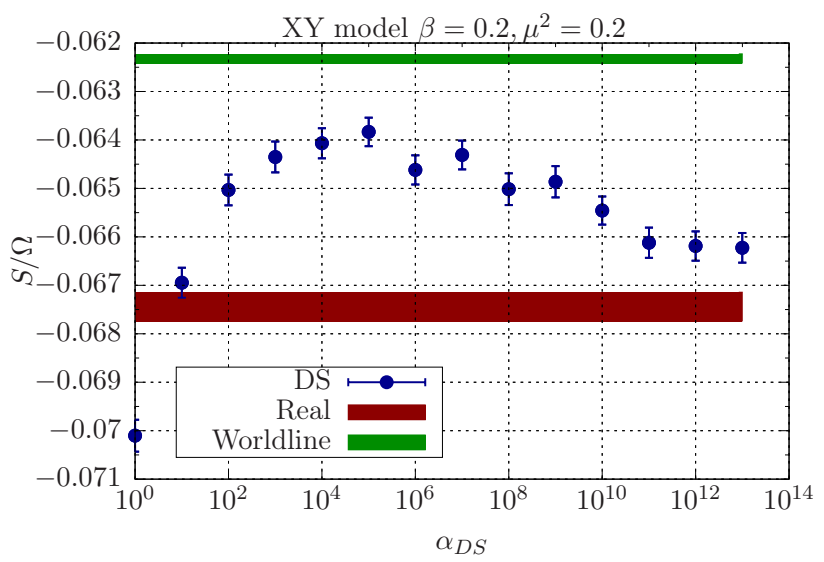

Figure 6. The action density $S / \Omega$ as a function of the control parameter $\alpha_{D S}$ for the XY model. The result for the worldline formulation (green) has been taken from [17].

control parameter $\alpha_{D S}$ for the largest $\mu$ studied here. It is obvious that even the best choice does not reproduce the correct result from the dual representation, shown in green. For very large $\alpha_{D S}$ the result approaches, as expected, the results from real Langevin simulations, shown in red. The source of the discrepancy between are DS and the world-line formulation, can be manifold. Our simulations are not extrapolated in terms of the step size correction, but some first evidence indicate that even smaller step sizes do not reconcile the observed deviation. Further definitions in terms of the DS forces are currently being studied and other formulations might show a better convergence.

\section{Conclusion and Outlook}

We presented an update on applications of the method of Dynamic Stabilisation. We studied the effects on the drifts appearing in complex Langevin simulation of HDQCD. We find that the additional force is improving the distribution of the drift. In particular, the non-SU(3) forces becomes more localised. However, a formal justification for DS is still in progress. For now, the method remains a heuristic procedure to cure instabilities occurring in complex Langevin simulations, which have been seen in simulations with smaller gauge couplings. We further illustrate an application of DS on the threedimensional XY model with finite chemical potential. We find a clear improvement towards the correct value, which is obtainable with a sign-problem-free dual representation. However, DS does not solve the discrepancy entirely. Especially for larger chemical potentials a small but significant difference remains. In future works we will explore the possibility of improved definitions for the DS force in the XY model.

\section{Acknowledgements}

We are indebted to Gert Aarts, Dénes Sexty, Erhard Seiler and Ion-Olimpiu Stamatescu for invaluable discussions and collaboration. We are grateful for the computing resources made available by HPC Wales. We acknowledge the STFC grant ST/L000369/1. B.J. was supported by the Schweizerischer Nationalfonds (SNF) under grant 200020-162515. F.A. is grateful for the support through the Brazilian government programme "Science without Borders" under scholarship number BEX 9463/13-5. 


\section{References}

[1] G. Aarts, I.O. Stamatescu, JHEP 09, 018 (2008), 0807. 1597

[2] D. Sexty, Phys. Lett. B729, 108 (2014), 1307.7748

[3] G. Aarts, E. Seiler, D. Sexty, I.O. Stamatescu, Phys. Rev. D90, 114505 (2014), 1408. 3770

[4] D.K. Sinclair, J.B. Kogut, PoS LATTICE2015, 153 (2016), 1510. 06367

[5] K. Nagata, J. Nishimura, S. Shimasaki (2016), 1606.07627

[6] G. Parisi, Y.s. Wu, Sci. Sin. 24, 483 (1981)

[7] G. Parisi, Phys. Lett. B131, 393 (1983)

[8] J.R. Klauder, Acta Phys. Austriaca Suppl. 25, 251 (1983)

[9] J.R. Klauder, J. Phys. A16, L317 (1983)

[10] J.R. Klauder, Phys. Rev. A29, 2036 (1984)

[11] E. Seiler, D. Sexty, I.O. Stamatescu, Phys. Lett. B723, 213 (2013), 1211. 3709

[12] G. Aarts, L. Bongiovanni, E. Seiler, D. Sexty, I.O. Stamatescu, Eur. Phys. J. A49, 89 (2013), 1303.6425

[13] G. Aarts, F. Attanasio, B. Jäger, E. Seiler, D. Sexty, I.O. Stamatescu, Acta Phys. Polon. Supp. 8, 405 (2015), 1506.02547

[14] G. Aarts, F. Attanasio, B. Jäger, D. Sexty, JHEP 09, 087 (2016), 1606.05561

[15] G. Aarts, F. Attanasio, B. Jäger, E. Seiler, D. Sexty, I.O. Stamatescu, AIP Conf. Proc. 1701, 020001 (2016), 1412.0847

[16] G. Aarts, F. Attanasio, B. Jäger, D. Sexty, Acta Phys. Polon. Supp. 9, 621 (2016), 1607.05642

[17] G. Aarts, F.A. James, JHEP 08, 020 (2010), 1005 . 3468

[18] P.H. Damgaard, H. Hüffel, Phys. Rept. 152, 227 (1987)

[19] A. Mollgaard, K. Splittorff, Phys. Rev. D88, 116007 (2013), 1309. 4335

[20] K. Splittorff, Phys. Rev. D91, 034507 (2015), 1412.0502

[21] J. Nishimura, S. Shimasaki, Phys. Rev. D92, 011501 (2015), 1504.08359

[22] J. Greensite, Phys. Rev. D90, 114507 (2014), 1406.4558

[23] G. Aarts, E. Seiler, D. Sexty, I.O. Stamatescu, JHEP 05, 044 (2017), 1701.02322

[24] F. Attanasio, B. Jäger, PoS LATTICE2016, 053 (2016), 1610. 09298

[25] G. Aarts, E. Seiler, I.O. Stamatescu, Phys. Rev. D81, 054508 (2010), 0912.3360

[26] G. Aarts, F.A. James, E. Seiler, I.O. Stamatescu, Eur. Phys. J. C71, 1756 (2011), 1101.3270

[27] S. Chandrasekharan, PoS LATTICE2008, 003 (2008), 0810. 2419

[28] D. Banerjee, S. Chandrasekharan, Phys. Rev. D81, 125007 (2010), 1001.3648 